\title{
On The Estimation of Parameters for the Inverse Weibul Distribution
}

\author{
Ahmed A. El - Sawy Eldesoky E. Afify
}

Faculty of Eng. Minufiya Univ. Shebeen El-Koom- Egypt.

Key Words: Inverse Weibull distribution, linear programming, Newton Raphson method, Reliability function.

\begin{abstract}
:
In this paper, we use the least absolute deviation and Newton Raphson methods, to estimate the parameters of inverse Weibull (IW) distribution, also we compare between these methods by using mean absolute percentage error of the reliability function, numerical example is given to clarify the use of these methods.
\end{abstract}

\section{Introduction:}

The two parameters inverse Weibull distribution

$$
f(t)=B \alpha^{-B} t^{-B-1} \exp \left[-1 /(\alpha t)^{B}\right] \quad t \geq 0 \quad \alpha, B>0
$$

is used as a model in the analysis of life testing data, it is also suitable model to describe mechanical degration phenomena. The probability of failure before time $t$ is given by the inverse Weibull commutative distribution function defined by:

$F(t)=\exp \left[-1 /(\alpha t)^{B}\right]$

\footnotetext{
Manuscript received from Dr. EL- DESOKYE. AFIFY on : $15 / 7 / 2000$ Accepted on : $1 / 8 / 2000$

Engineering Research Bulletin, Vol 23,No 4, 2000 Minufiya University, Faculty of Engineering, Shebien EI-Kom, Egypt, ISSN 1110-1180
} 
In R. Calabria and Pulcini (1989) the statistical properties of the ML estimators of the parameters $\beta$ and $\alpha$, and the reliability function $R(t)$ for a complete I.W. sample have been investigated. Tables of lower confidence limits for $R(t)$ have been provided. In P. Erto (1989) the estimators for $\beta, \alpha$ and $\mathrm{R}(\mathrm{t})$ obtained by using the plotting positions technique, also the $\mathrm{L}$. S. estimators and their statistical properties have been provided. R. Calabria and least squares methods to estimate the inverse Weibull distribution parameters. In Calabria and Pulcini (1994), Bayes prediction intervals are derived, both when no prior information is available and when prior information on the unreliability level at a fixed time is introduced.

\section{Least absolute deviation method, (LAD):}

The reliability function of $(1)$ is given by:

$$
\begin{aligned}
& R(t)=1-e^{-(\alpha t)^{-\beta}} \\
& =1-e^{-\theta t^{-\beta}} \\
& e^{-\theta^{-\beta}}=1-R(t)
\end{aligned}
$$

Taking the logarithm of both sides of (4)

$\operatorname{Ln} \theta-B \operatorname{Ln} t=\operatorname{Ln}(-\operatorname{Ln}[1-R(t)])$

Or:

$\operatorname{Ln} t=\frac{\operatorname{Ln} \theta}{\beta}-\frac{1}{\beta} \operatorname{Ln}(-\operatorname{Ln}[1-R(t)]$

$y_{i}=B_{0}+B_{1} X_{i}$

Where $y_{i}=\operatorname{Ln} t_{i}, \quad B_{\circ}=\frac{\operatorname{Ln} \theta}{\beta} \quad$ and $\quad B_{1}=\frac{-1}{\beta}$

And $\quad x i=\operatorname{Ln}(-\operatorname{Ln}[1-R(t)]), \quad \theta=\alpha^{-\beta}$

Consider the linear regression problem in the form of $n$ observations $\left(\mathrm{y}_{\mathrm{i}}, \mathrm{X}_{\mathrm{i}}\right)$, for $\mathrm{i}=1,2, \ldots \ldots \ldots, \mathrm{n}$. the regression model is given by (5), where $\mathrm{B}_{0}$ and $\mathrm{B}_{1}$ 
are the parameters, the problem is to find $\hat{\beta}_{\mathrm{o}}$ and $\hat{\beta}_{1}$ estimations of $\beta_{\mathrm{o}}$ and $\beta_{1}$ so that the dependent variables $y_{i}$ cause predicted as

$\hat{\mathbf{y}}_{\mathbf{i}}=\widehat{\boldsymbol{\beta}}_{\mathbf{0}}+\widehat{\beta}_{\mathbf{1}} \mathbf{X}_{\mathbf{i}}$

Also, the problem is to find the values of the parameters $\beta_{o}$ and $\beta_{i}$, which will minimize the least absolute deviations (LAD) so that the problem is

$\operatorname{Min} . \sum_{i=1}^{n}\left|y_{i}-\left(\beta_{o}+\beta_{1} x_{i}\right)\right|$

Problem (7) have been transferred to linear programming formulation by Charnes, Cooper and Ferguson (1955), so the problem (7) is equivalent to:

Minimize $\sum_{i=1}^{n}\left(P_{i}+N_{i}\right)$

Subject to

$\beta_{0}+\beta_{i} X_{i}+P_{i}-N_{i}=y_{i}, \quad i=1,2, \ldots, n$.

$\mathrm{P}_{\mathrm{i}} \geq 0, \quad \mathrm{~N}_{\mathrm{i}} \geq 0, \quad \mathrm{i}=1,2, \ldots, \mathrm{n}$.

Where $\mathrm{P}_{\mathrm{i}}, \mathrm{N}_{\mathrm{i}}$, are the positive and negative errors and the problem is to find the values of $\beta_{o}$ and $\beta_{1}$ that minimize the errors.

The standard tableau form of this problem is shown in the table (1)

Table (1): The LAD problem in tableau form

\begin{tabular}{|c|c|c|c|c|c|c|c|c|}
\hline $\mathrm{P} 1$ & $\mathrm{P} 2$ & $\mathrm{Pn}$ & $\mathrm{N} 1$ & $\mathrm{~N} 2 \ldots$. & $\mathrm{Nn}$ & $\mathrm{B} 0$ & $\mathrm{~B} 1$ & $\mathrm{RHS}$ \\
\hline 1 & & & -1 & & & 1 & $\mathrm{X} 1$ & $\mathrm{Y} 1$ \\
\hline & 1 & & & -1 & & 1 & $\mathrm{X} 2$ & $\mathrm{Y} 2$ \\
\hline & & & & & & $\vdots$ & $\vdots$ & $\vdots$ \\
\hline & & & & & & $\vdots$ & $\mathrm{Xn}-1$ & $\mathrm{Yn}-1$ \\
\hline & & 1 & & & $1-$ & 1 & $\mathrm{Xn}$ & $\mathrm{Yn}$ \\
\hline 1 & 1 & 1 & 1 & 1 & 1 & 0 & 0 & $\mathrm{Z}(\min )$ \\
\hline
\end{tabular}




\section{Newton Raphson method (NR):}

The Newton Raphson method is one algorithm for minimizing the sum of the residuals between data and nonlinear equations Newton's method for system uses the nxn Jacobian matrix in the vector situation and substitute by the derivative with the inversion of the Jacobian matrix, this method for finding the solution $\theta$ to the nonlinear system of equations represented by the vector equations.

$F(x)=0, \quad$ has the form

$\theta^{k}=\theta^{k-1}-[J(\theta) k-1]^{-1} F\left(\theta^{k-1}\right)$

For $\mathrm{K} \geq 1$

Given the initial approximation $\theta^{(\mathrm{o})}$ to the solution $\theta$, the initial.values of $\theta$ can be assumed to be zero. Where $J(t)$ is the first order derivative of $R(t)$ in the matrix form given by:

$$
\mathrm{J}(\mathrm{t})=\left[\begin{array}{ll}
\frac{\partial R_{1(t)}}{\partial \alpha} & \frac{\partial R_{i}(t)}{\partial \beta} \\
\frac{\partial R_{2}(t)}{\partial \alpha} & \frac{\partial R_{2}(t)}{\partial \beta}
\end{array}\right]
$$

$$
\mathrm{R}(\mathrm{t})=1-e^{-(\alpha t)^{-8}}
$$

$$
\begin{aligned}
& \frac{\partial R(t)}{\partial \alpha}=B \alpha^{-B-t} t^{-B} e^{-(\alpha t)^{-t}} \\
& \frac{\partial R(t)}{\partial \alpha}=\ln (\alpha t)(\alpha t)^{-B} e^{-(\alpha t)^{-i}}
\end{aligned}
$$

and $\frac{\partial R(t)}{\partial a_{k}}$ is the particle derivative of the function $\mathrm{R}(\mathrm{t})$ with respect to the $\mathrm{K}^{\mathrm{th}}$ parameter evaluated at the $i^{\text {th }}$ data point.

To compute the accuracy of estimates of parameters and reliability function we use the mean absolute percentage error (MAPE) defined by 
$\mathrm{MAPE}=\frac{1}{n} \sum\left|Z_{t}\right|$

Where

$$
\mathrm{Z}_{\mathrm{t}}=\left(\frac{R(t)-R_{e}(t)}{R(t)}\right) * 100
$$

And

$R(t)$ is actual reliability function. $R_{e}(t)$ is the estimated reliability function

\section{Numerical Example:}

Consider the following 10 simulated data from an inverse Weibull distribution with parameters $\alpha=0.01$ and $\beta=2$ listed in the following table (4)

Table (1):

\begin{tabular}{|c|c|c|c|c|}
\hline 55.3 & 66.9 & 87.0 & 112.5 & 118.4 \\
\hline 129.1 & 141.5 & 167.1 & 245.4 & 335.5 \\
\hline
\end{tabular}

The maximum likelihood estimates are $\hat{\alpha}=0.0102$ and $\hat{\beta}=2.152$

The values of $x_{i}$ and $y_{i}$ in equation (6) are given by the table (2)

Table (2)

\begin{tabular}{|c|c|}
\hline$x$ & $y$ \\
\hline 1.18 & 4.01 \\
\hline 0.8 & 4.2 \\
\hline 0.28 & 4.47 \\
\hline-0.24 & 4.72 \\
\hline-0.34 & 4.77 \\
\hline-0.51 & 4.86 \\
\hline-0.69 & 4.95 \\
\hline-3.01 & 6.11 \\
\hline-1.8 & 5.5 \\
\hline-2.42 & 5.82 \\
\hline
\end{tabular}

The corresponding tableau of the tableau (1) is given by 
Table (3);

\begin{tabular}{|c|c|c|c|c|c|c|c|c|}
\hline$\overline{\mathrm{P} 1}$ & P2 & $\mathrm{Pn}$ & $\mathrm{N} 1$ & $\mathrm{~N} 2 \ldots \ldots$ & $\mathrm{Nn}$ & $\mathrm{B} 0$ & B1 & RHS \\
\hline 1 & & & -1 & & & 1 & 1.18 & 4.01 \\
\hline & 1 & & & -1 & & 1 & 0.8 & 4.2 \\
\hline & & & & & & 1 & 0.28 & 4.47 \\
\hline & & & & & & 1 & -0.24 & 4.72 \\
\hline & & 1 & & & $1-$ & 1 & -0.34 & 4.77 \\
\hline & & & & & & 1 & -0.51 & 4.86 \\
\hline & & & & & & 1 & -0.69 & 4.95 \\
\hline & & & & & & 1 & -3.01 & 6.11 \\
\hline & & & & & & 1 & -1.8 & 5.5 \\
\hline & & & & & & 1 & -2.42 & 5.82 \\
\hline 1 & 1 & 1 & 1 & 1 & 1 & 0 & 0 & $Z(\min )$ \\
\hline
\end{tabular}

The values of $\beta_{0}$ and $\beta_{1}$ are computed with the aid of the computer program (1), then $\hat{\alpha}$ and $\hat{\beta}$ can be obtained.

The estimates $\hat{\alpha}, \hat{\beta}$ from Newton Raphson method are obtained after 4 iteration by the computer program (2), as shown in table (4).

The reliability estimates $R_{1}, R_{2}, R_{3}$ and $R_{4}$, are computed from relation (3) at different values of the estimated parameters $\hat{\alpha}$ and $\hat{\beta}$, while MAPE is computed from the relation (8), as shown in table (5)

Table (4) The Parameters Estimates

\begin{tabular}{|c|c|c|c|c|}
\hline Method & \multicolumn{2}{|c|}{ No outliers } & \multicolumn{2}{c|}{ Outliers } \\
\hline & $\alpha$ & $\beta$ & $\alpha$ & $\beta$ \\
\hline LAD & 0.0102 & 19912 & 0.0101 & 1.9952 \\
\hline NR & 0.1553 & 1.704 & 0.1668 & 1.811 \\
\hline
\end{tabular}


Table (5) Reliability Estimates

\begin{tabular}{|c|c|c|c|c|c|}
\hline \multirow[t]{2}{*}{$\mathrm{T}$} & \multirow[t]{2}{*}{ True $\mathrm{R}(\mathrm{t})$} & \multicolumn{4}{|c|}{$R(t)$} \\
\hline & & $\mathrm{R} 1$ & $\mathrm{R} 2$ & $\mathrm{R} 3$ & $\mathrm{R} 4$ \\
\hline 1 & 0.96199 & 0.95616 & 0.02530 & 0.95910 & 0.01773 \\
\hline 2 & 0.89294 & 0.88239 & 0.01835 & 0.88766 & 0.01259 \\
\hline 3 & 0.73318 & 0.71876 & 0.01177 & 0.72592 & 0.00784 \\
\hline 4 & 0.54621 & 0.53210 & 0.00761 & 0.53931 & 0.00493 \\
\hline 5 & 0.51000 & 0.49681 & 0.00698 & 0.10336 & 0.00410 \\
\hline 6 & 0.45119 & 0.43903 & 0.00602 & 0.44107 & 0.00384 \\
\hline 7 & 0.39313 & 0.38221 & 0.00516 & 0.38765 & 0.00326 \\
\hline 8 & 0.30102 & 0.04697 & 0.00389 & 0.04759 & 0.00241 \\
\hline 9 & 0.15300 & 0.14862 & 0.00202 & 0.11083 & 0.00120 \\
\hline 10 & 0.08101 & 0.08270 & 0.00119 & 0.08387 & 0.00068 \\
\hline & & 2.2423 & 98.42947 & 1.1176 & 98.98219 \\
\hline
\end{tabular}

$R_{1}$ is Reliability estimates from LAD in the absence of outliers

$R_{2}$ is the reliability estimates from Newton Raphson method in the absence of outliers.

$R_{3}$ is reliability estimate from $L A D$ in the presence of outliers

$R_{4}$ is reliability estimates from NR in the presence of outliers.

\section{Conclusion:}

From table (4) the parameters estimates from least absolute deviation method are close to the true values and following table (5) the MAPE from least absolute deviation method are less than MAPE from Newton Raphson method. Also least absolute deviation method is easy to use, and takes little time on computer, also it is more accurate as compared with Newton Raphson method, because the MAPE from it is less than MAPE from the Newton Raphson method. 


\section{References}

1) Calabria, $R$ and Pulcini, G (1989) Confidence limits for reliability and tolerance limits in the inverse Weiball distribution Reliability Engineering and system safety $24,77-85$.

2) Calabria, R. and Pulcini G. (1990): On the maximum likelihood and least squares Estimation in the inverse Weibull distribution statistica Applicate Vol. 2, N.1.

3) Calabria $R$ and Pulcini, G (1994) Bayes 2 sample prediction for the inverse Weibull distribution Commun. Statist.- Theory Meth, 23(6), 1811 - 1824.

4) Charnes, A., W.W. Cooper and R. Ferguson (1955) optimal estimation of executive compensation by linear programming Management Sci. 1, 138-151.

5) Erto, P (1989). Genesi, Proprieta ED identificazione del Modello di Sopravvivenza Weibull inverso stutista, Applicata, Vol.1 N.2.

6) Paul Rech, Paul Schmidbaur and Jamie Eng. (1989) Least absolute regession revisited a simple labeling method for finding a lar line Commun. Statist. Simula., 18(3), $943-955$.

Computer program (1)

DIMENSION X(10), Y(10), B(10,10), A $(10,10) \operatorname{ELERR}(10,10)$

OPEN $(5$, FILE $=$ 'AM.DAT')

OPEN (6, FILE = 'AM.OUT')

$\mathrm{N}=10$

$\left.\operatorname{READ}\left(5,{ }^{*}\right), X(I), Y(\mathrm{l}), \mathrm{I}=1, \mathrm{~N}\right)$

DO $10 \mathrm{~J}=\mathrm{J}+1, \mathrm{~N}$

IF (X(I).EQ X(J)) THEN

$\mathrm{B}(\mathrm{I}, \mathrm{J})=100000.0$

ELSE

$\mathrm{B}(\mathrm{I}, \mathrm{J})=(\mathrm{Y}(\mathrm{J})-\mathrm{Y}(\mathrm{I})) \mathrm{V}(\mathrm{X}(\mathrm{J})-\mathrm{X}(\mathrm{I}))$

ENDIF

$\mathrm{A}(\mathrm{I}, \mathrm{J})=\mathrm{Y}(\mathrm{I})-\mathrm{B}(\mathrm{L}, \mathrm{J}) * \mathrm{X}(\mathrm{I})$

SUM $=0.0$

DO $30 \mathrm{~K}=1, \mathrm{~N}$

$30 \mathrm{SUM}=\mathrm{SUM}+\mathrm{ABS}(\mathrm{Y}(\mathrm{K})-\mathrm{A}(\mathrm{I}, \mathrm{J})+\mathrm{B}(\mathrm{I}, \mathrm{J}) * \mathrm{X}(\mathrm{K})))$

$\operatorname{ELERR}(\mathrm{I}, \mathrm{J})=\mathrm{SUM}$

10 CONTINUE

$\mathrm{L}=1$

$\mathrm{M}=2$ 


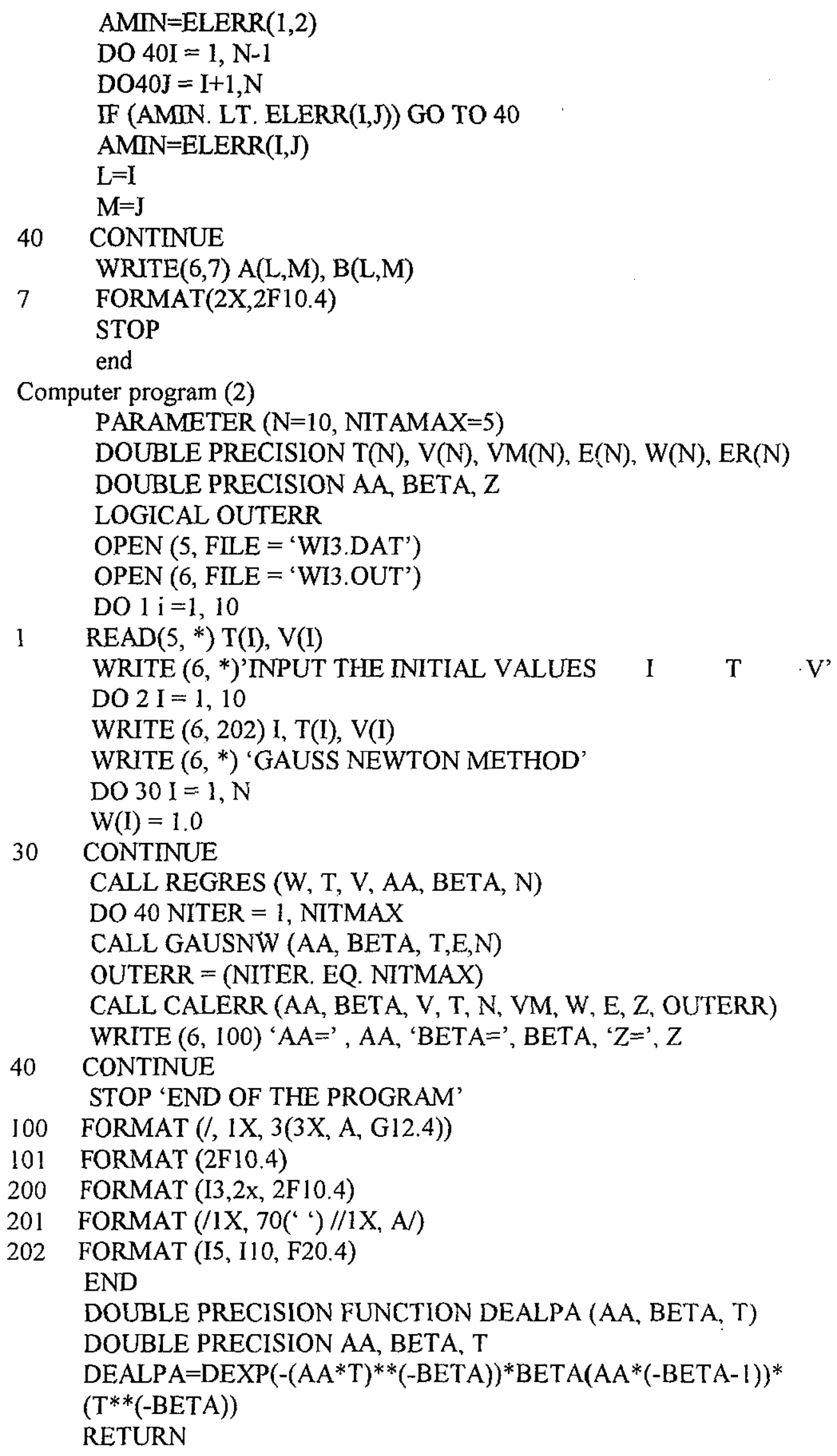




\section{END}

DOUBLE PRECISION FUNCTION DEBETA (AA, BETA, T)

DOUBLE PRECISION AA, BETA, T

DEBETA $=$ DEXP $\left(-\left(\mathrm{AA}^{*} \mathrm{~T}\right) * *(-\mathrm{BETA})\right)^{*} \mathrm{DLOG}(\mathrm{AA} * \mathrm{~T})^{*}(\mathrm{AA} * \mathrm{~T}) * * *(\mathrm{BETA})$

RETURN

END

SUBROUTINE GAUSNW (AA, BETA, T, E, N)

DOUBLE PRECISION AA, BETA, T(N), E(N)

DOUBLE PRECISION DELTAA, DELTAB, DET

DOUBLE PRECISIO a(2,2), B(2)

INTEGER $\mathrm{N}$

$\mathrm{I}=\mathbf{I}+\mathbf{1}$

DO $11 \mathrm{~J}=1,2$

$\mathrm{B}(\mathrm{J})=0.0$

DO $11 \mathrm{~K}=1,2$

$\mathrm{A}(\mathrm{J}, \mathrm{K})=0.0$

11

CONTINUE

$\mathrm{I}=\mathrm{I}+1$

DO $20 \mathrm{I}=1, \mathrm{~N}$

$\mathrm{A}(1,1)=\mathrm{A}(1,1)+$ DEALPA $(\mathrm{AA}, \mathrm{BETA}, \mathrm{T}(\mathrm{I})) * * 2$

$\mathrm{A}(1,2)=\mathrm{A}(1,2)+\mathrm{DEALPA}(\mathrm{AA}, \mathrm{BETA}, \mathrm{T}(\mathrm{I}))^{*} \mathrm{DEBETA}(\mathrm{AA}, \mathrm{BETA}, \mathrm{T}(\mathrm{I}))$

$\mathrm{A}(2,2)=\mathrm{A}(2,2)+\mathrm{DEBETA}(\mathrm{AA}, \mathrm{BETA}, \mathrm{T}(\mathrm{I}))^{* * 2}$

$\mathrm{B}(1)=\mathrm{B}(1)+\mathrm{DEALPA}(\mathrm{AA}, \mathrm{BETA}, \mathrm{T}(\mathrm{I}))^{* *} \mathrm{E}(\mathrm{I})$

$\mathrm{B}(2)=\mathrm{B}(2)+\mathrm{DEBETA}(\mathrm{AA}, \mathrm{BETA}, \mathrm{T}(\mathrm{I}))^{*} \mathrm{E}(\mathrm{I})$

20 CONTINUE

$\mathrm{DET}=\mathrm{A}(1,1) * \mathrm{~A}(2,2)-\mathrm{A}(1,2) *(1,3)$

DELTAA $=-(\mathrm{A}(1,2) * \mathrm{~B}(2)-\mathrm{A}(2,2) * \mathrm{~B}(1)) / \mathrm{DET}$

$\mathrm{DELTAB}=(\mathrm{A}(1,1) * \mathrm{~B}(2)-\mathrm{A}(1,2) * \mathrm{~B}(1)) / \mathrm{DET}$

$\mathrm{AA}=\mathrm{AA}+$ DELTAA

$\mathrm{BETA}=\mathrm{BETA}+\mathrm{DELTAB}$

RETURN

END

SUBROUTINE CALLERR (AA,BETA, V,T,N,V,M,W,E,Z,OUTERR)

PARAMETER (NMAX $=10)$

LOGICAL OUTERR

$\mathrm{N}=10$

DOUBLE PRECISION AA, BETA, T(N), E(N)

DOUBLE PRECISION V(N), W(N), VM(N), ER(NMAX),Z

INTEGER N,I

IF (N.GT.NMAX) STOP 'CALERR; INCREASE NMAX VALUE'

IF (OUTERR) WRITE $(*, 100)$

$Z=0.0$

DO $12 \mathrm{I}=1, \mathrm{~N}$

$\mathrm{VM}(\mathrm{I})=\operatorname{DEXP}\left(-(\mathrm{AA} T(\mathrm{I}))^{*} \mathrm{BETA}\right)$

$E(I)=V(I)-V M(I)$

$\mathrm{ER}(\mathrm{I})=\mathrm{E}(\mathrm{l}) / \mathrm{V}(\mathrm{I})$

$\mathrm{Z}=\mathrm{Z}+\mathrm{E}(\mathrm{l}) / \mathrm{V}(\mathrm{I})$

$\mathrm{Z}=\mathrm{Z}+\mathrm{E}(\mathrm{I}) * * 2$ 
12

100

200

IF (OUTERR) WRITE $(6,200) \mathrm{T}(\mathrm{I}), \mathrm{V}(\mathrm{I}), \mathrm{VM}(\mathrm{I}), \mathrm{E}(\mathrm{I}), \mathrm{ER}(\mathrm{I})$

\section{CONTINUE}

FORMAT (/T11, 'T', T25, 'V', T39, 'VM', T10, 'E', T65, 'ER')

FORMAT (3F14.4, 2E15. 4)

RETURN

END

SUBROUTINE REGRES (W, T, V, AA, BETA, N)

PARAMETER $($ NMAX $=10)$

LOGICAL OUTERR

$\mathrm{N}=10$

DOUBLE PRECISION AA, BETA

DOUBLE PRECISION V(N), T(N), W(N)

DOUBLE PRECISION A(2,2), B(2), C(2), DET

INTEGER N, I

$\mathrm{A}(1,1)=0.0$

$\mathrm{A}(1,2)=0.0$

$\mathrm{A}(2,2)=0.0$

$\mathrm{B}(1)=0.0$

$\mathrm{B}(2)=0.0$

DO $13 \quad \mathrm{I}=1, \mathrm{~N}$

$\mathrm{A}(1,1)=\mathrm{A}(1,1)+\mathrm{W}(\mathrm{I})$

$\mathrm{A}(1,2)=\mathrm{A}(1,2)+\mathrm{W}(\mathrm{I}) * \mathrm{DLOG}(\mathrm{T}(\mathrm{I}))$

$\mathrm{A}(2,2)=\mathrm{A}(2,2)+\mathrm{W}(\mathrm{I}) * \operatorname{DLOG}(\mathrm{T}(\mathrm{I}))^{* * 2}$

$\mathrm{B}(1)=\mathrm{B}(1)+\mathrm{W}(\mathrm{I}) * \operatorname{DLOG}(\mathrm{DLOG}(1 / \mathrm{V}(\mathrm{I})))$

$\mathrm{B}(2)=\mathrm{B}(2)+\mathrm{W}(\mathrm{I}) * \mathrm{DLOG}(\mathrm{DLOG}(1 / \mathrm{V}(\mathrm{I}))) * \mathrm{DLOG}(\mathrm{T}(\mathrm{I}))$

13 CONTINUE

$\mathrm{DET}=\mathrm{A}(1,1) * \mathrm{~A}(2,2)-\mathrm{A}(1,2) * \mathrm{~A}(1,2)$

$\mathrm{C}(1)=\left(\mathrm{B}(1)^{*} \mathrm{~A}(2,2)-\mathrm{B}(2)^{*} \mathrm{~A}(1,2)\right) / \mathrm{DET}$

$\mathrm{C}(2)=\left(\mathrm{B}(2) * A(1,1)-\mathrm{B}(1)^{*} \mathrm{~A}(1,2)\right) / \mathrm{DET}$

$\mathrm{BET} \mathrm{T}=\mathrm{C}(2)$

$\mathrm{AA}=\mathrm{DEXP}(\mathrm{C}(1) / \mathrm{C}(2))$

RETURN

END 


\section{ملـفم البــثث}

\section{تقدير معالم توزيع وابيل العكسى}

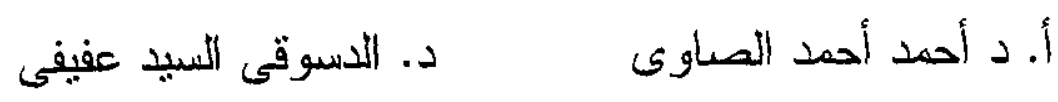

كلية الهندسة - شبين الكوم - جامعة المنوفية

فـى هذا البحثث تم استخدام طريقة أقــــل الانحر افــات المطلقــة

وطريقة نيونت رافسون لتقدير معالم توزيع و ابيل العكنى فـــى حالـــة

وجود نقط شاذة وفى حاللة عدم وجود نقط شاذة وقد تم عمل مقارنة بين

هذه الطرق فى الحالثين وتنين الآتى من خلال منال عددى:

1- باستخدام طريقة أقل الانحر افات المطلقة فإن قيم المعالم تكــــون

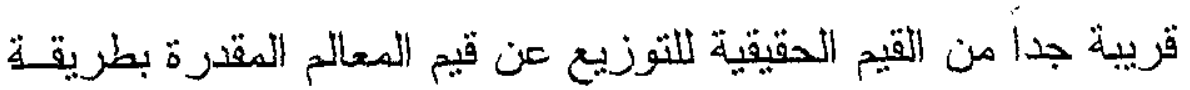

نيونن ر افسون.

r- طريقة أقل الاتحر افات المطلقة تأخذ وقت قليل على الكمبيونــر

عن طريقة نيونن رلفسون.

ب- متوسط الخطأ المئوى المطلق لدالة الاعتمادية في طريقة أقـــلـ

الانحر افات أقل منه فى طريقة نيونن رافسون خاصة في وجود نقط

شاذة. 